\title{
El ruido en la ciudad neerlandesa
}

\author{
Adriana de Ruiter \\ Becaria en la Cátedra de Derecho Administrativo de la \\ Facultad de Derecho de la Universidad de León
}

Sumario: I. NOTA PRELIMINAR. II. SISTEMA GENERAL DE LA LEY GENERAL DE MEDIO AMBIENTE Y DE LA LEY DE LAS MOLESTIAS ACÚSTICAS. 2.1. Potestades de las Administraciones Públicas. 2.2. Objetivo y aplicación de las dos Leyes relevantes. 2.3. Los Instrumentos de la LMA. 2.3.1. Zonas. 2.3.2. Aparatos. 2.4. Los instrumentos de la LGMA; licencias. 2.5. Tráfico Aéreo. 2.6. Mantenimiento administrativo. III. EL CIUDADANO EN EL DERECHO ADMINISTRATIVO. 3.1. Participación en la toma de decisiones. 3.2. Recursos administrativos. 3.3. Recursos contencioso-administrativos. IV. DERECHO CIVIL. 4.1. El juez civil como juez supletorio. 4.2. El acto ilícito y los vecinos. V. CONSIDERACIÓN FINAL.

\section{NOTA PRELIMINAR}

Desde hace aproximadamente dos décadas está desarrollándose a gran velocidad la legislación medioambiental, tanto por parte de la Unión Europea, como por parte de las autoridades nacionales y regionales. Mediante la adopción de numerosas leyes, reglamentos, directivas y decisiones se pretende frenar las consecuencias nocivas que ha tenido el crecimiento económico e industrial en el entorno natural, conforme al principio de un crecimiento sostenible '. Si bien en un principio la atención se centraba sobre todo en una protección del medio ambiente contra la contaminación más palpable procedente sobre todo de la industria, cada vez se concentra más en tipos de contaminación más ligera, y procedente también de la vida cotidiana, entre ellos el ruido, que es precisamente el tema de investigación de este trabajo.

Antes de poder comenzar el desarrollo de la materia es preciso hacer hincapié en algunas características del Derecho medioambiental. Desde el principio de la lucha contra la contaminación la política medioambiental se caracterizaba por ser una política meramente sectorial. Es decir, no existía un plan general de principios, ni normas generales que tuvieran como fin la protección general del medio ambiente, sino que se regulaba cada tipo de contaminación por separa-

1 Este principio fue desarrollado por primera vez en el Informe Brundtland, y ha sido recogido en numerosos tratados internacionales y en la legislación nacional. Ejemplo de su aplicación encontramos en el art. 2 Tratado de la Comunidad Europea, que dispone que la Comunidad tiene por misión promover (...) un crecimiento sostenible y no inflacionista que respete el medio ambiente (...). 
do. Lógica consecuencia de esta política sectorial era el traslado de problemas de un sector a otro. Así, por ejemplo, son famosos los casos en los que para evitar la contaminación del aire, se llevaba a cabo una limpieza con el agua, y mediante la posterior limpieza del agua se causaba contaminación del suelo. También en la materia de nuestro interés se han producido los casos en que las medidas de protección contra la contaminación acústica causaban por ejemplo contaminación paisajística.

La legislación relativa a la contaminación acústica se caracteriza por el desarrollo de dos vías de protección. La primera vía consta de la regulación concerniente a aparatos causantes de ruidos, como son los vehículos motorizados, todo tipo de maquinaria y cualquier otra fuente móvil de ruido. La segunda vía de protección se lleva a cabo a través de medidas protectoras del receptor del ruido, en la mayoría de los casos viviendas y edificios.

En este trabajo se analizará la solución encontrada, o en vías de ser hallada, en los Países Bajos. En este país, famoso por su sobrepoblación, el ruido puede ser considerado uno de los problemas más cotidianos. En parte, se trata aquí del ruido procedente de polígonos industriales y las vías de transporte, así como de los centros de ocio en las ciudades. Estos tipos de contaminación acústica han sido objeto de una amplia legislación administrativa. Pero muy destacable en un país tan pequeño y con una población muy densa, son los problemas entre los vecinos. Claramente sintomático al respecto es el dato de que, en los Países Bajos, después de los divorcios, la mayoría de los casos ante los tribunales civiles trata precisamente de conflictos entre vecinos por culpa de sus animales domésticos, sus instrumentos musicales y sus aparatos domésticos.

Para ofrecer una noción general de la legislación neerlandesa, se divide este trabajo en tres apartados. En el primero se tratará la legislación administrativa medioambiental, donde se encuentra la base de la lucha contra el ruido. Después se analizarán los recursos administrativos y contencioso-administrativos de que disponen los ciudadanos para protegerse a título particular, en el caso de que la Administración pública no lo haga. El último apartado estará dedicado al Derecho civil, que también ofrece soluciones a problemas ruidosos.

\section{SISTEMA GENERAL DE LA LEY GENERAL DE MEDIO AMBIENTE Y DE LA LEY DE LAS MOLESTIAS ACÚSTICAS}

\subsection{Potestades de las Administraciones públicas}

Sin querer tratar a fondo el problema de la atribución de competencias, es preciso dedicar unas palabras a la potestad ejecutiva que las Leyes medioam- 


\section{EL RUIDO EN LA CIUDAD NEERLANDESA}

bientales atribuyen a las Administraciones públicas. En este marco se deben distinguir tres niveles, siendo el primero el correspondiente al Gobierno central, el segundo a la provincia y, por último, el correspondiente al poder municipal ${ }^{2}$.

El órgano central encargado de la ejecución de la legislación medioambiental es el Ministerio de Vivienda, Ordenación del Territorio y Protección del Medio Ambiente (VROM) ${ }^{3}$. Este Ministerio asume también las competencias relativas a la preparación y el establecimiento de los Tratados Internacionales, así como de las leyes medioambientales. El Ministro tiene entre sus tareas la de llevar a cabo una política ambiental y controlar que otros órganos sigan las directrices procedentes de su Ministerio.

Obviamente pueden encontrarse en el nivel central otros Ministerios que se verán enfrentados con los problemas medioambientales. En los casos en que existan fricciones entre las políticas desarrolladas por los diferentes Ministerios, será preciso la coordinación de actividades para evitar conflictos de competencia. En muchas leyes encontramos disposiciones obligando a esta coordinación.

La Ley General de Medio Ambiente (LGMA) ${ }^{4}$ concede el papel protagonista a la Provincia. Es éste el nivel donde se ejecuta la política medioambiental y en el cual se desarrollan las normas adicionales. Uno de los instrumentos indicados para esta labor es el Plan de Zona, mediante el cual la Provincia puede establecer normas para su desarrollo futuro.

Si anteriormente el Ayuntamiento jugaba un papel primordial en la protección medioambiental, y sobre todo en las normas relativas al ruido, últimamente se aprecia una disminución de la importancia del Municipio en favor de la Provincia. La tarea de la que se encarga el Ayuntamiento se reduce prácticamente a la ejecución de normas procedentes de las Administraciones superiores. No obstante, en el tema de la contaminación acústica, las labores del Ayuntamiento son aún considerables. El Ayuntamiento es quien lleva a cabo y desarrolla los planes urbanísticos que, como veremos más adelante, establecen indirectamente los niveles de ruido en cada rincón del municipio. Además, el Ayuntamiento lleva a cabo la política de vivienda, mediante la cual puede, con

2 Ya que los Países Bajos conocen una estructura estatal centralista, la atribución de competencias en materias legislativas no suele causar mayores problemas. Es el legislador central el encargado de desarollar la legislación cualesquiera que sea su tema. Dentro del marco establecido por el legislador central, los legisladores locales disponen de cierta autonomía para regular los temas según convenga. Desde luego, tanto el legislador central como los locales deben mantenerse dentro de los límites establecidos por la Unión Europea.

3 Volkshuisvesting, Ruimtelijke Ordening en Milieu.

4 Wet Milieubeheer de 1993. 
la ayuda de los instrumentos de la Ley de Vivienda ${ }^{5}$, conceder licencias de obras, o dictar órdenes de demolición de viviendas y edificios. Además de la ejecución, el Ayuntamiento se encarga en gran medida del mantenimiento de la legislación.

\subsection{Objetivo y aplicación de las dos Leyes relevantes}

La protección acústica está regulada en dos Leyes: la Ley General de Medio Ambiente (LGMA) y la Ley de las Molestias Acústicas (LMA) ${ }^{6}$.

La LGMA es la heredera directa de la Ley de Molestias ${ }^{7}$ de 1875 , que fue derogada por la LGMA en 1993, y de la Ley de Disposiciones Generales sobre Higiene Ambiental ${ }^{8}$ de 1980, en la cual se agrupaban normas relativas a la uniformización de la participación de los ciudadanos, los recursos y la coordinación de los procedimientos relativos a las licencias. Con la entrada en vigor de la LGMA fueron derogadas varias leyes sectoriales, integrando toda la legislación medioambiental en una sola Ley. Este proceso de integración, aún no concluido, se ha estacionado de momento en la ampliación en 1993 de la LGMA a las reglas concernientes a instalaciones, los planes para la política ambiental y algunas normas cualitativas medioambientales. También se ha añadido un capítulo sobre el mantenimiento de las normas medioambientales.

Con esta Ley se marca un hito en la historia de la protección medioambiental en los Países Bajos. Se quiere lograr una mejor protección medioambiental mediante una política integral, evitando así el traslado de problemas de un sector a otro. Además, se ha intentado conseguir cierta claridad en la legislación, lo que no sólo es más cómodo para el ciudadano, sino también para la misma Administración. O, como el Gobierno lo ha formulado, un mejor instrumento legal puede conseguir un medio ambiente de más calidad. Desdichadamente, esto no quiere decir que ya no existan otras leyes sectoriales que, por sus características, no se prestan para su regulación en la LGMA. No obstante, la LGMA debe ser considerada la clave en la protección medioambiental.

Consecuencia de esto es que se debe entender la LMA dentro del marco de la LGMA, considerando siempre la LMA como lex specialis relativa a la LGMA. Según el preámbulo de la LMA, ésta tiene como objetivo la protección

\footnotetext{
5 Woingwet.

6 Wet Geluidshinder.

7 Hinderwet.

8 Algemene Bepalingen Milieuhygiëne.
} 
EL RUIDO EN LA CIUDAD NEERLANDESA

integral del medio ambiente y de la sanidad pública contra el ruido. Sin embargo, no se aplica la LMA a tráfico aéreo ni al interior de instalaciones, ya que al primer causante de ruido se le aplica la Ley del Tráfico Aéreo (LTA) ${ }^{9}$ y al segundo la Ley de Condiciones Laborales ${ }^{10}$.

Un gran jurista neerlandés, el Dr. Donner, ha tipificado la LMA como un gran monumento legislativo. Por desgracia suya, y nuestra, veremos que esta Ley se está pareciendo más a un castillo en ruinas que a un ejemplo de eficacia y perfección burocrática, ya que de sus 186 artículos, nada menos que 84 están derogados. Se plantea por lo tanto la duda de si esta Ley puede cumplir su objetivo, ya que numerosos temas han sido regulados en la LGMA. El resultado es que se aplica la Ley únicamente a los ruidos procedentes de ciertos aparatos y a la problemática de las zonas industriales y las zonas de tráfico rodado.

\subsection{Los instrumentos de la LMA}

\subsubsection{Zonas}

La LMA aplica la zona como un instrumento para proteger las viviendas y edificios del ruido procedente de carreteras y vías de ferrocarril, incluyendo el tranvía y el metro, y el ruido procedente de polígonos industriales. Ambas zonas estarán integradas en el plan urbanístico que deben establecer los correspondientes municipios.

El Capítulo VI LMA regula las zonas alrededor de las carreteras, y establece parámetros que definen los límites aceptables de ruido dentro de la zona. Diferencia en primer lugar entre zonas dentro y fuera del caso urbano, dejando claro que dentro de la ciudad se acepta un nivel superior de ruido y una menor amplitud de la zona. También el número de vías y la velocidad máxima permitida en la carretera determinan el nivel de ruido aceptable. Así, por ejemplo, las carreteras de velocidad máxima de $30 \mathrm{~km}$. por hora no precisan de la instalación de una zona, ya que a esta velocidad los coches prácticamente no causan ruido. Esta velocidad máxima coincide con la de los barrios residenciales. Un tercer factor determinante es la constatación por el Pleno del Ayuntamiento de que los niveles de ruido no sobrepasen los $50 \mathrm{db}(\mathrm{A})$ a 10 metros de la carretera. Si esto es así, no se instalará una zona, según el art. 74 de la LMA. Otro parámetro determinante para los niveles de ruido es la diferencia entre situaciones nuevas y existentes, habiendo reglas más estrictas en el primer supuesto.

\footnotetext{
9 Luchtvaartwet.

Arbeidsomstandighedenwet.
} 
Según los arts. 82-86 de la LMA, el máximo nivel aceptable de ruido en el frontal de las viviendas será de $50 \mathrm{db}(\mathrm{A})$. Sin embargo, si los niveles de ruido superan este umbral será necesario que el Ayuntamiento obtenga el permiso de la Provincia, a la vez que debe proponer planes para mejorar la situación y adecuarla a los niveles permitidos.

Las normas relativas a las zonas alrededor del ferrocarril, el metro y el tranvía se encuentran en los arts. 105-106 LMA. En los supuestos del tráfico de ferrocarril la Ley no establece los límites, sino que delega esta potestad en el Gobierno, labor que el Gobierno llevó a cabo aprobando el Reglamento de Molestias Acústicas de Ferrocarril " ${ }^{11}$ en 1987. El sistema de este Reglamento es muy parecido al previsto para las carreteras en la LMA, con la diferencia de que el límite normal es de $60 \mathrm{~dB}(\mathrm{~A})$, ya que se espera que a largo plazo las tecnologías aplicadas al ferrocarril serán de mejor calidad y disminuirán el ruido ${ }^{12}$.

Otras zonas que deben establecerse afectan a las instalaciones industriales o empresariales. Aquí ya se aprecian las complicaciones que causa el hecho de que la LMA es una lex specialis relativa a la LGMA. Las instalaciones están reguladas también por esta última, a cuyas reglas más adelante nos tendremos que referir. Ahora es suficiente mencionar que ambas Leyes delegan en el Gobierno la potestad para indicar qué instalaciones precisan de una zona ${ }^{13}$, o en el caso de la LGMA, una licencia. A diferencia de las zonas alrededor de carreteras, estas zonas estarán fijadas por el Ayuntamiento en el plan urbanístico y no derivan ipso iure de la Ley.

Al igual que para las zonas de las carreteras, se diferencia entre zonas nuevas y zonas existentes, permitiendo más ruido en las zonas ya existentes. En las zonas nuevas se permiten niveles de $50 \mathrm{~dB}(\mathrm{~A})$ en los frontales de las casas dentro de las zonas, y para las zonas viejas el nivel tope es $55 \mathrm{~dB}(\mathrm{~A})$, según el art. 46 y art. 65 LMA. En ambos casos será posible que la Provincia conceda permiso excepcional para ampliar estos límites a 55 y $65 \mathrm{~dB}(\mathrm{~A})$, para zonas nuevas y ya existentes, respectivamente, siempre que el nivel de ruido dentro de las casas no sobrepase los 35 y $40 \mathrm{~dB}(\mathrm{~A})$. La Dirección municipal está obligada a desarrollar un plan de demolición o de saneamiento de las viviendas afectadas por las excepciones, que precisa la autorización de la Provincia y del

11 Besluit geluidhinder spoorwegen, 25 de marzo de 1987.

12 Tanto para las zonas de carreteras como de ferrocarril se utiliza la técnica de "paredes de ruido", que pueden ser grandes pantallas, arbolado o pequeñas colinas de tierra al lado de la zona, para evitar que el ruido salga de la zona. Sin embargo, este método ha sido sumamente criticado, ya que causa contaminación paisajística.

Art. 41 de la LMA. 
Ministro de VROM, teniendo en cuenta que nunca se podrá sobrepasar el máximo de $65 \mathrm{~dB}(\mathrm{~A})$ en los frontales de las viviendas, según versa el art. 72 LMA.

\subsubsection{Aparatos}

Además de las zonas, la LMA regula la base para que el Gobierno pueda tomar medidas relativas a aparatos y al ruido que éstos produzcan. Esta base permite al Gobierno prohibir o limitar mediante licencias o normas de calidad el fabricar, importar, tener en provisión, vender, entregar, trasladar o usar determinados aparatos, según los arts. 2 a 8 de la LMA. Estos artículos han sido utilizados entre otros para asimilar las medidas impuestas por el Derecho Comunitario, regulando la contaminación acústica que causan entre otros los vehículos motorizados (Reglamento de 30 de noviembre de 1981), las motocicletas (Reglamento de 15 de agosto de 1985), y las máquinas de construcción (Reglamento de 21 de febrero de 1994).

\subsection{Los instrumentos de LGMA; licencias}

La clave del régimen impuesto por la LGMA es la obligación de obtener una licencia para poner en funcionamiento una instalación. Como hemos visto anteriormente, esta Ley pretende integrar toda la política ambiental. Por lo tanto, se concede una licencia medioambiental general para todo tipo de actividades, sea cual sea la contaminación que cause. De tal suerte, una actividad que cause diferentes tipos de contaminación, sólo precisará de una licencia medioambiental, sin perjuicio de la obligación de solicitar las demás licencias, como la autorización del proyecto $u$ otros permisos que no sean meramente ambientales. La principal ventaja que ofrece este sistema es que se permite a las autoridades públicas imponer reglas concretas en cada caso particular, o como lo dicen los alemanes, Gesetzgebung für den Einzelfall. En la resolución de la licencia se podrá tener en cuenta, además de los datos peculiares del medio ambiente en que se piensa encuadrar la instalación, la opinión pública sobre el proyecto.

Por instalación se entiende cualquier actividad humana profesional, o no profesional pero sí de volumen o dimensiones importantes, que se lleve a cabo dentro de un cierto terreno con cierta frecuencia. Los dos criterios decisivos para determinar si una actividad constituye o no una instalación han sido desarrollados en la jurisprudencia procedente de una Ley anterior ${ }^{14}$. El primero trata la profesionalidad, término interpretado de manera extensiva, de modo que

14 La Ley de Molestias de 1875, anteriormente citada. 
un hobby de una persona puede precisar también la licencia medioambiental si alcanza dimensiones elevadas ${ }^{15}$. El segundo criterio es la localidad fija ${ }^{16}$. Además, la instalación no sólo comprende actuaciones, sino también conjuntos de aparatos, construcciones e incluso productos. El art. 1.1.4 LGMA delega la potestad al Ministro de VROM para establecer más categorías de actividades que podrán ser consideradas una instalación.

$\mathrm{El}$ art. 8.1 LGMA distingue entre dos tipos de instalaciones. Las primeras caen bajo el régimen del art. 8.1 que prohíbe el fundar, cambiar o mantener una instalación sin la debida licencia. Pero la LGMA exceptúa de esta obligación las instalaciones a las que según el art. 8.40.1 se aplican las normas generales del Gobierno. Este último artículo estipula que el Gobierno puede desarrollar categorías de instalaciones que, si cumplen las reglas establecidas para ellas, no precisarán la licencia ambiental. No obstante, el hecho de que no se necesite la licencia, no libra a los promotores de la actividad del deber de notificar esta actividad al órgano encargado. El Reglamento correspondiente del Gobierno indicará cuál es este órgano. Este sistema de normas generales ha causado una importante disminución de funciones en la labor del Ayuntamiento, ya que con anterioridad a estas reglas el Ayuntamiento concedía cada licencia individualmente, con el resultado de que en 1985 se otorgaron alrededor de $400.000 \mathrm{li}$ cencias, y este número descendió en los últimos años a sólo unas $100.00{ }^{17}$. Uno de los campos que abarcan las normas generales es el de la hostelería.

No obstante, quedan muchos casos en los que será necesaria la licencia. Conforme a lo previsto en el art. 1.1.3 LGMA, el Gobierno ha establecido todas las categorías en el Reglamento Medioambiental de Instalaciones y Licencias ${ }^{18}$, siendo este Reglamento aplicable tanto a la LGMA, como a la LMA ${ }^{19}$. Este Reglamento contiene tres Anexos, en los cuales se establecen diferentes categorías que precisan de licencias y se indica cuáles son los órganos competentes. La regla general es que, según la magnitud del proyecto, un órgano de mayor o menor peso será el responsable de resolver la licencia solicitada.

En el Reglamento también se establecen los requisitos necesarios para la solicitud de la licencia, pero las demás reglas concernientes al procedimiento

is Sentencia de 8 de abril de 1975.

16 Sentencia de 25 de noviembre de 1959.

17 De esta suerte se quiere conseguir además la llamada «retirada de la Administración» de la actividad empresarial y comercial.

18 Inrichtingen en Vergunningenbesluit, 5 de enero de 1993.

19 El mismo Reglamento también ejecuta más leyes sectoriales que aún no están integradas en la LGMA. 
se encuentran en otras Leyes. La LGMA incluye en su capítulo 13 normas relativas al procedimiento, pero éstas deben ser consideradas lex specialis relativas a la Ley General de Derecho Administrativo (LGDA) ${ }^{20}$. La LGMA declara de aplicación los apartados 3.5.2 hasta 3.5.5 de la LGDA. En estos artículos la LGDA estipula un procedimiento extensivo en el cual la opinión pública tiene un grado de decisión importante.

Las Administraciones públicas encargadas de formular la resolución de la solicitud no poseen total discrecionalidad. Sólo se puede negar la licencia medioambiental por razones medioambientales ${ }^{21}$, según el art. 8.10 LGMA. Existen dos tipos de criterios por los que se rige la resolución. El primer criterio consta de las normas pseudo-legales, es decir, todo tipo de directrices, convenios y normas políticas. La jurisdicción contencioso-administrativa aplica estas normas mediante el principio de la seguridad jurídica ${ }^{22}$. El segundo criterio es puramente legal. No se puede otorgar una licencia si fuese contraria al Derecho. Los arts. 8.8 hasta 8.10 LGMA hacen mención de normas legales que se deben tener en cuenta en la resolución de la licencia, entre ellas, las normas válidas derivadas de la LMA, sin limitar en gran medida la discrecionalidad de la Administración. En cambio, otras normas tendrán un efecto decisivo sobre la resolución, como son el estado del medio ambiente, las consecuencias que la actividad tendrá sobre ella, el Plan Medioambiental en vigor, etc. Esta vinculación de la Administración pública a la legislación y a las normas pseudo-legales implica que el juez —en su caso- podrá ejercer un control bastante amplio, ya que se ve restringida la potestad discrecional de la Administración Pública.

El contenido de la licencia esta formulado en el art. 8.11.1 LGMA, dejando claro que además de normas adicionales relativas a la protección medioambiental, la solicitud también forma o debe formar parte de la licencia. Esta exigencia tiene como fin evitar que la Administración varíe de una u otra manera la solicitud y se otorgue una licencia no solicitada. Las normas adicionales que pueden ser parte de la licencia sólo son admitidas si las normas generales en vigor relativas a la materia en cuestión así lo permiten, y, desde luego, las nòr-

20 Este Ley se puede comparar un poco a la española Ley 30/1992, de Régimen Jurídico de las Administraciones Públicas y del Procedimiento Administrativo Común, de 26 de noviembre, aunque la LGDA abarca también, como veremos más adelante, el procedimiento contencioso-administrativo.

21 En la mayoría de los casos, el que solicita la licencia medioambiental precisará también otras licencias o autorizaciones. Desde luego puede suceder que se le niege la licencia en otro procedimiento por razones que no tengan nada que ver con la protección medioambiental.

22 Esto no quiere decir que mediante el principio de la seguridad jurídica se puede ir en contra de los intereses medioambientales, pero los tribunales sí tienen en cuenta este principio a la hora de valorar las alegaciones de los ciudadanos ante la Administración. 
REALA 274-275 (MAYO-DICIEMBRE, 1997)

mas generales prevalecen sobre las normas adicionales, en caso de que éstas sean contrarias a las primeras.

El último aspecto a tratar en relación con las licencias es su naturaleza y su relación con las zonas. La licencia puede ser definida como un acto real, es decir, no se otorga a una persona, sino a una actividad. Esto implica que si se traspasa la actividad a un tercero, éste no tendrá que solicitar de nuevo las licencias necesarias. Además, la licencia constituye un acto individual, lo cual, como veremos más adelante, es uno de los requisitos para poder recurrir las decisiones de la Administración pública. En cuanto a la relación con las zonas, es menester recordar que la LMA es una lex specialis relativa a la LGMA, lo que implica la prevalencia de la LMA sobre la LGMA. En este caso concreto este hecho lleva consigo que si aún no han sido fijadas las zonas, no se podrá otorgar la licencia prevista en la LGMA.

\subsection{Tráfico aéreo}

La regulación concerniente a los aeropuertos y el tráfico aéreo no está en la LMA, sino en la Ley de Tráfico Aéreo (LTA). Aunque el objetivo principal de esta Ley es la seguridad aérea, desde 1978 se han ido introduciendo también artículos concernientes a la contaminación acústica.

La LTA establece para medir los niveles de ruido un sistema complejo, de unidades de costes, permitiendo un cálculo que toma en consideración diversos factores, como el número anual de pasajeros, niveles máximos de ruido durante el pasaje, total de pasajes al año y un factor extra que «penaliza» el ruido nocturno. En el art. 2 LTA se considera el nivel de ruido factor determinante de la aeronavegabilidad de los aviones, coincidiendo en el art. 4 con la prohibición de utilizar un avión que no cumple las condiciones de aeronavegabilidad.

Otro problema concerniente a los ruidos causados por los aviones es la ubicación del aeropuerto. Según la LTA, el Ministro de Transportes debe, en cooperación con su compañero del Ministerio de Medio Ambiente, indicar qué terrenos serán utilizados para la construcción o ampliación de aeropuertos. Además deben participar en la toma de decisiones los municipios y otros cuerpos públicos afectados, incluyéndose también municipios fuera de los Países Bajos, como afirmó el Tribunal de La Haya ${ }^{23}$. Con la indicación del terreno, será preciso establecer una zona, fuera de la cual no se podrán sobrepasar ciertos niveles de ruido. El Pleno municipal integrará esta zona en el plan urbanís-

23 Sentencia de 3 de mayo de 1990, en este caso concreto se trató tanto de los municipios belgas como de los alemanes. 
EL RUIDO EN LA CIUDAD NEERLANDESA

tico. Aunque contra las alteraciones de los planes urbanísticos los ciudadanos poseen el derecho a objetar, no es así en el caso de las indicaciones para los aeropuertos. La razón de esta exclusión es que en la toma de decisiones sobre la ubicación de aeropuertos ya ha habido muchas posibilidades de participación, tanto por parte de los municipios, como por parte de los ciudadanos.

No obstante lo previsto en la legislación, aún no se ha llevado a cabo el establecimiento de la zona alrededor de Schiphol ${ }^{24}$, ni de otros aeropuertos más pequeños. El problema de las zonas es que, además de que definirlas cuesta dinero, posteriormente y conociendo los resultados, la dirección del aeropuerto en cuestión se verá obligada a tomar las medidas protectoras establecidas en la LTA. Como las cuestiones económicas no pueden justificar el no cumplimiento de la Ley, esta anomalía ha sido condenada por el Presidente del Tribunal de la Haya ${ }^{25}$, que dio en 1992 orden a la Dirección de Schiphol de comenzar la definición de la zona inmediatamente. Por suerte para los vecinos de los aeropuertos, el Tribunal Supremo ${ }^{26}$ ha dejado claro que la existencia de zonas y medidas protectoras no les impide solicitar una indemnización al juez conforme al Derecho civil, pero sobre este punto volveremos más adelante.

\subsection{Mantenimiento administrativo}

Como bien se sabe, pocos ciudadanos, sea en la vida laboral o en la vida privada, cumplen las Leyes por gusto propio, de tal suerte que las Leyes que no disponen de medios de control, son buenas para atiborrar bibliotecas, pero poco eficaces para tener cierta incidencia sobre el comportamiento de la ciudadanía. Conscientes de este hecho, los poderes legislativos han introducido también un capítulo sobre el mantenimiento en la LGMA ${ }^{27}$. En este capítulo, el 18 , se han integrado varios instrumentos ya existentes en las Leyes sectoriales anteriores a la LGMA. Así, también, el art. 148 LMA declara que para el mantenimiento de la misma, se aplicarán las normas de la LGMA.

Según versa el art. 18.2 LGMA el órgano encargado de llevar a cabo el control y en su caso ejercer potestades de penalización, es el mismo órgano que debe resolver las licencias, o sea, en la mayoría de los casos el Ayuntamiento o la Provincia. Este órgano tiene la obligación de registrar los datos concernientes a la instalación, tratar y contestar las denuncias y llevar un con-

\footnotetext{
24 El aeropuerto de Amsterdam.

25 Sentencia de 15 de octubre de 1992.

26 Sentencia de 26 de noviembre de 1993.

27 ¡Sin perjuicio de las disposiciones en el Código Penal!
} 
trol administrativo. La naturaleza de estas obligaciones es dudosa, y se plantea la pregunta de si los ciudadanos disponen del derecho subjetivo a que la Administración pública realmente cumpla con sus labores. La legislación no impone de qué manera, con qué frecuencia, ni siquiera qué consecuencias debe tener la actuación administrativa. Esto hace pensar que se trata aquí de potestades discrecionales, y es difícil sostener que los ciudadanos tengan un derecho subjetivo a una protección efectiva por parte de la Administración. Aunque los tribunales suelen imponer a la Administración una responsabilidad más extensiva, no podrán imponer las medidas que deba tomar la Administración.

El art. 18.5 contiene una referencia a las facultades otorgadas a los funcionarios encargados del control. Esta enumeración limitativa es necesaria para evitar fricciones con el principio de la legalidad, ya que los funcionarios en cuestión no forman parte del cuerpo de la policía, y precisan por lo tanto de una atribución especial para poder ejercer ciertas potestades de las cuales un ciudadano normal no dispone. Entre ellas encontramos el control sobre los libros y documentos de carácter empresarial, la investigación de los medios de transporte y su contenido, y la entrada con aparatos de medición en donde juzguen necesario, siempre que no sean viviendas.

El capítulo 18 dota de tres instrumentos específicos a la Administración pública para que pueda actuar con cierta eficacia. Éstos son la coacción administrativa, la multa administrativa y la revocación de la licencia o el permiso.

Por la coacción se entiende la facultad de la Administración de adecuar una situación ilegal a la legislación vigente, a costa del infractor. Tanto el Ayuntamiento como la Provincia ya disponen de esta potestad sobre la base de los arts. 125 Ley de Ayuntamiento y 122 de la Ley de la Provincia. Sin embargo, la introducción de este instrumento fue precisa, ya que el Ministro, también encargado en ciertos casos del control, no dispone de una base general. Ejemplo de cómo dar uso a este instrumento es la posibilidad de paralizar el funcionamiento de una instalación, sea parcial o completamente.

El uso de la coacción no precisa previa intervención del juez, pero sí es necesario que la Administración advierta al infractor con anterioridad al ejercicio de la coacción. Este aviso o notificación constituye un acto, que se puede recurrir.

El segundo instrumento mencionado en la LGMA es la multa coercitiva. Es menester diferenciar entre esta multa coercitiva, que tiene su base en el art. 18.9 LGMA y la multa coercitiva que en ocasiones puede imponer el juez civil. En el primer caso se trata claramente de un instrumento en manos de la Administración para, sin previa intervención de los jueces, sea cual sea su jurisdicción, 
EL. RUIDO EN LA CIUDAD NEERLANDESA

intentar imponer a un infractor el cumplimiento de las Leyes. La multa coercitiva no podrá utilizarse arbitrariamente, sino solamente cuando no perjudique intereses medioambientales, ya que este instrumento, si bien cambia la situación legal, no altera por sí mismo el comportamiento del infractor.

El último instrumento, y el más grave para el infractor, es la revocación de la licencia, tal como queda formulado en el artículo 18.12 LGMA. Se puede utilizar cuando el infractor no actuó conforme a la licencia, no respetó las normas generales o infringió las normas relativas a la licencia. No obstante, el art. 18.12.3 declara que se debe otorgar un tiempo de recuperación al infractor.

Lo que se ha dicho de la multa coercitiva, también se puede aplicar a la revocación de la licencia, es decir, tampoco altera la situación fáctica. Ambos instrumentos sólo podrán ser eficaces si, en caso de que el infractor no cambie su comportamiento, la Administración use también la coacción administrativa.

\section{EL CIUDADANO EN EL DERECHO ADMINISTRATIVO}

En este apartado, se prestará atención a las posibilidades de participación y a los recursos existentes en el Derecho Administrativo neerlandés, para que los ciudadanos puedan influir en la toma de decisiones o combatir una decisión de la Administración. Trataremos tanto la posición del afectado por las decisiones de la Administración como la posición de los terceros.

\subsection{Participación en la toma de decisiones}

Habitualmente se considera al ciudadano la parte débil en el proceso administrativo, afirmación que no puede ser negada si observamos las amplias potestades, discrecionales en su mayor parte, de la Administración pública. Se tiende a dar mucha importancia a los recursos, pero tan importantes como ellos pueden ser las garantías de participación que están surgiendo en las legislaciones de muchos países, así también en los Países Bajos.

El procedimiento aplicable a la resolución de una solicitud de licencia según la LGMA, contiene un momento importante en el cual el ciudadano puede hacer objeciones concernientes a la resolución propuesta por el órgano competente ${ }^{28}$. El artículo 3.24 LGDA otorga este derecho a todos, incluyendo desde luego al titular del proyecto. Sin embargo, el art. 3.26 LGDA ofrece la posibili-

28 Este procedimiento está escrito en al apartado 3.5 de la LGDA, y se llama el procedimiento de preparación extensiva, aplicable a las licencias medioambientales según el art. 13 LGMA. 
dad a la Administración pública de reducir este círculo de personas, no pudiendo afectar nunca esa reducción a los propios interesados. El interesado es la persona cuyo interés está directamente involucrado en la decisión, o sea, el que tenga un interés propio, directo y objetivamente determinable en el asunto concreto.

El procedimiento para establecer las zonas es distinto, ya que, como hemos visto, las zonas alrededor de las carreteras derivan directamente de la LMA, no dejando lugar a que los vecinos del municipio participen en la toma de decisiones. Pero tampoco la zona industrial deja mucho hueco a la participación de la ciudadanía. Razón de esta exclusión es que las zonas se establecen dentro del marco del plan urbanístico, y el procedimiento mediante el cual se proyecta el plan urbanístico sí posee un momento de participación pública. De esta suerte el ciudadano sólo puede indirectamente hacer objeciones contra las zonas que se quieran instalar.

Una acción peculiar la encontramos en el capítulo del mantenimiento administrativo de la LGMA. El art. 18.14 dispone que cada individuo podrá solicitar a la Administración competente la aplicación de las medidas de control y de puesta en marcha de los instrumentos estudiados en el párrafo 2.6. En sí esto no es nada nuevo, ni siquiera muy necesario, ya que a los habitantes de los Países Bajos se les reconoce el derecho de petición en el art. 5 de la Constitución. No obstante, según los tribunales el art. 18.14 concede el derecho a una respuesta de la Administración, aunque no necesariamente positiva, pero siempre conforme a las normas establecidas para la actuación administrativa, como son los principios de motivación de los actos, la prohibición de desviación de poder, etc. ${ }^{29}$. Esta respuesta constituye un acto que, como veremos, podrá ser recurrido, sea en un procedimiento administrativo, sea en un procedimiento contencioso-administrativo.

\subsection{Recursos administrativos}

Al igual que el Derecho español, el Derecho neerlandés también obliga a los ciudadanos a interponer primeramente un recurso administrativo antes de poder recurrir ante los tribunales. Los recursos están regulados en la LGDA, y mediante el art. 20.1 LGMA se estipula que contra las decisiones tomadas sobre la base de la LGMA y algunas leyes sectoriales, como la LMA, se aplicarán las disposiciones de la LGDA.

29 Ver por ejemplo la Sentencia de 24 de diciembre de 1993 del Presidente del Departamento de Conflictos del Consejo del Estado. 
En este recurso la Administración pública tiene la oportunidad de reflexionar sobre su decisión, sin que el ciudadano se vea obligado a recurrir a métodos más complejos, como es el de recurrir ante los tribunales. De este modo se evitará además la congestión de asuntos en los tribunales, mal que parecen padecer la mayoría de los tribunales europeos.

El resultado de la existencia de la actio popularis como hemos visto en el párrafo anterior es que habrá más interesados, ya que cada persona que haya hecho objeciones en el proceso de tomar las decisiones relativas a la primera petición, tendrá derecho a recurrir la decisión. Ahora bien, la otra cara de este derecho es que los que no hayan mostrado ningún tipo de interés durante la toma de decisiones, tampoco podrán posteriormente alegar la decisión tomada, incluso si se trata del titular del proyecto.

El recurso se interpondrá ante el mismo órgano que haya tomado la primera decisión, según el art. 6.4 LGDA. Para la resolución del recurso, la Administración podrá llevar a cabo un control, tanto de la legalidad como de la oportunidad, gozando así de la posibilidad real de reflexionar si la decisión tomada ha sido la más adecuada, bien sea por razones legales, o por razones oportunas. En el procedimiento de resolución, la Administración tendrá que oír una vez más las alegaciones de todos los interesados.

Como último aspecto mencionaremos aquí que los afectados por las decisiones de control administrativo, como hemos visto en el párrafo 2.6, también podrán interponer recursos alegando la no legalidad o no oportunidad del uso de los instrumentos administrativos.

\subsection{Recursos contencioso-administrativos}

En muchas ocasiones será preciso, y afortunadamente posible, que el ciudadano recurra ante los tribunales en búsqueda de una solución.

Conforme al art. 20.1 de la LGMA el interesado puede interponer un recurso contra una decisión tomada sobre la base del procedimiento de preparación extensiva. La legitimación necesaria para ser admitido depende de si el demandante en cuestión ha hecho o no objeciones en el momento adecuado, es decir, durante la participación pública en el procedimiento administrativo. Se hace una excepción para aquellas personas que por razones ajenas a su voluntad no hayan podido participar, pero esta excepción se interpretará de manera restrictiva. Así, por ejemplo, no se considera que el estar de vacaciones sea suficiente razón para no presentarse en la auditoría pública. 
REALA 274-275 (MAYO-DICIEMBRE, 1997)

Otro criterio a tener en cuenta para valorar la legitimación de los demandantes es el principio de la relatividad, procedente del Derecho privado. Si un ciudadano alega verse perjudicado por el no cumplimiento de las normas por la Administración pública, no será admitido si la norma infringida no estaba prevista para proteger los intereses del ciudadano en cuestión.

El art. 20.13 LGMA abre además la posibilidad para los interesados a recurrir las decisiones que no sean tomadas mediante el procedimiento de la preparación extensiva. Esta categoría puede abarcar un gran abanico de decisiones, como son las decisiones concernientes a la aplicación de sanciones, las normas específicas complementarias a las normas generales, la solicitud de una indemnización, etc.

Además de la legitimación del interesado, habrá que estudiar si la decisión tomada constituye un acto recurrible. La misma LGMA excluye de la posibilidad de recurrir los actos a un número de decisiones. Esto no quiere decir que contra los últimos no cabe ningún recurso, sino que son recurribles mediante otra Ley o procedimiento.

El Derecho meramente administrativo también excluye determinados actos de la posibilidad de ser recurridos. Así, la LGDA excluye de manera general las normas vinculantes de alcance general y las normas políticas. Tampoco las actuaciones basadas en el Derecho privado se podrán recurrir ante los tribunales contencioso-administrativos. La siguiente excepción, más importante, se produce cuando no se trata de un acto, formulado como tal en la LGDA ${ }^{30}$. Esta exclusión se presenta entre otros en los supuestos de actuaciones materiales. Así, por ejemplo, la decisión de proceder a la coacción constituye un acto recurrible, pero no la ejecución de la misma.

Se ha visto ya que la LGDA parte de la idea de que primero habrá de interponerse un recurso administrativo. No obstante, se considera que en casos en que se ha utilizado el procedimiento de la preparación extensiva, se podrá recurrir directamente a los tribunales, ya que en este supuesto la Administración ha tenido tiempo y oportunidades de reflexionar sobre su decisión, y no es de esperar que la cambie en otro procedimiento. En el tema que tratamos, este dato implica que el recurso administrativo no será preciso en numerosos conflictos, ya que las resoluciones medioambientales más importantes se toman a través de este procedimiento.

30 Los requisitos para que sea un acto en el sentido de la LGDA son: una decisión de voluntad escrita procedente de un órgano administrativo y basada en el Derecho administrativo que constituye una actuación jurídica. Se diferencia entre actos de alcance general y actos de alcance individual, art. 1.3 LGDA. 
EL RUIDO EN LA CIUDAD NEERLANDESA

Otro dato peculiar en los conflictos medioambientales es que el juez competente no es el mismo que en los demás casos contencioso-administrativos. Según la LGDA, se debe presentar el recurso ante la Sala de lo contencioso-administrativo de los tribunales de primera instancia con la posibilidad de apelación ante el Departamento de Jurisdicción contencioso-administrativa del Consejo de Estado. Pero el art. 20.1 LGMA envía los conflictos medioambientales directamente a este Departamento, que debe juzgar en primera y última instancia, dejando penosamente palpable la falta de protección jurisdiccional en los asuntos medioambientales. Esta laguna tiene sus raíces en el hecho de que se está reorganizando la estructura jurisdiccional, y se espera que este problema será solucionado en breve.

Además de poder recurrir los actos, los recurrentes disponen de la posibilidad de solicitar medidas provisionales para evitar que se cometan daños irreparables, como postulan tanto el art. 20.3 LGMA, como el art. 8.81 LGDA. Es el Presidente del tribunal el competente para decidir sobre estas medidas, y no dudará en aplicarlas si los intereses implicados así lo exigen. En algunos casos no es ni siquiera necesario que el Presidente pondere los intereses, sino que se impone imperativamente si hay intereses ecológicos importantes o costes económicos muy altos.

Ahora bien, antes de cerrar este apartado es importante señalar un último detalle. El control llevado a cabo por el juez es desde luego un control de legalidad. Sin embargo, se puede apreciar desde hace un tiempo que el juez intenta controlar cada vez más las facultades discrecionales de la Administración Pública, mediante la aplicación de los principios generales de Derecho.

\section{DERECHO CIVIL}

Hemos visto que las posibilidades de que dispone el ciudadano para recurrir las decisiones de la Administración ante la jurisdicción contencioso-administrativa dependen de si esa decisión constituye un acto y si el ciudadano en cuestión está legitimado para recurrirlo. En aquellos supuestos en que se hace palpable una laguna en la protección de los ciudadanos, será posible recurrir ante los tribunales civiles. Pero esto no es todo. En numerosas ocasiones, el Derecho civil, además de ofrecer la protección jurisdiccional, puede ofrecer soluciones materiales adecuadas para los problemas de contaminación acústica.

\subsection{El juez civil como juez supletorio}

En todos aquellos supuestos en que los tribunales de lo contencioso-administrativo no son competentes para conocer los casos, el juez civil puede asu- 
mir la competencia. Éste será el caso en las actuaciones administrativas de hecho, las actuaciones normativas y las directrices políticas. Obviamente también las actuaciones basadas en el Derecho privado podrán ser recurridas ante los tribunales civiles, de tal suerte que disminuyan las consecuencias negativas del hecho de que ante los tribunales de lo contencioso-administrativo la base del pleito es obligatoriamente el acto administrativo. De este modo pueden considerarse los tribunales civiles y los tribunales de lo contencioso-administrativo como vasos comunicantes, ya que si no tiene competencia uno, la asumirá el otro.

El Tribunal Supremo decidió ya en $1915^{31}$ que el juez civil es competente si la parte demandante basa su petición sobre un derecho subjetivo derivado del Derecho civil objetivo - la teoría del objectum litis-, aunque ambas partes pertenezcan a la Administración pública, como en el caso citado de 1915 en que se trataba de dos Ayuntamientos. En este sentido, es importante señalar que los jueces, aunque aún no exista un derecho general a un medio ambiente limpio, sí reconocen un derecho subjetivo a un entorno vital sano, protegido por el Derecho civil. Así, por ejemplo, a los vecinos del aeropuerto Schiphol se les reconoció el derecho a no tener que soportar los ruidos procedentes del mismo. En otro caso ${ }^{32}$, señero en la historia jurisdiccional de la protección medioambiental, el Tribunal Supremo declaró que los intereses medioambientales están protegidos por el art. 6:162 Código Civil (Cc.), artículo que dispone las normas relativas al acto ilícito, como veremos más adelante.

Ahora bien, esto no quiere decir que los ciudadanos o la Administración siempre tengan libertad para elegir el juez que más les convenga. El Tribunal Supremo sólo acepta la competencia del juez civil como juez supletorio si no existe otra vía jurisdiccional que esté revestida de suficientes garantías ${ }^{33}$. Aunque el juez superior no explicó qué garantías debe contener un procedimiento para que sea aceptable, en la doctrina sí encontramos criterios como la independencia de los jueces, la calidad del procedimiento, la audiencia, los plazos, etc.

Si bien el juez civil ofrece una protección a los ciudadanos y a la Administración, esto no quiere decir que exista una acción pública en el Derecho civil. Tal como en el procedimiento contencioso-administrativo, el Derecho civil también parte de la teoría de la legitimidad para poder recurrir ante los tribunales. La regla general en el Derecho civil es que sin interés no puede haber una

31 El conflicto se produjo entre los Ayuntamientos de Guldemond y de Noorwijkerhout, Sentencia de 31 de diciembre de 1915.

32 De Nieuwe Meer, Sentencia de 27 de junio de 1986.

33 Sentencia del Tribunal Supremo de 22 de febrero de 1957. 
acción. No es suficiente que sea un interés general, sino que debe tratarse de un interés real, personal e individual. Es menester en este marco hacer una referencia al principio de la relatividad, también conocido como la Schutznormtheorie. Un ciudadano no podrá tener acceso a una acción si las normas que según él habían sido infringidas no estaban escritas para defender sus intereses. Esta teoría trae como consecuencia que existan lagunas en la protección medioambiental, ya que muchas normas medioambientales pretenden defender intereses generales, de las cuales no se pueden derivar derechos subjetivos. Este caso se presentó cuando un vecino de un proyecto de viviendas recurrió a los tribunales alegando que en la licencia de construcción no había ninguna previsión sobre las normas de aislamiento, pero él no fue considerado «interesado», ya que para serlo se precisaba una relación especial con el proyecto, el ser vecino no justifico su legitimidad ${ }^{34}$.

Además de las personas naturales, las personas jurídicas también pueden intentar buscar soluciones en el Derecho civil. Por la Ley de 6 de abril 1994 se introdujeron en el Código civil los art. 3:305.a y 305.b, en los cuales se establece la legitimidad de las asociaciones defensoras de intereses generales para recurrir ante los tribunales, siendo esta ley la afirmación de la jurisprudencia ya asentada por el Tribunal Supremo. En el caso De Nieuwe Meer ${ }^{35}$, el Tribunal Supremo sentenció que una asociación precisará en primer lugar de una mención clara de su objetivo en su Estatuto, para tener la necesaria legitimidad. Pero la sola mención en los Estatutos no es el único factor determinante para ser admitido ante los tribunales, sino que la legitimidad también se demuestra a través de las circunstancias concretas. Así sentenció el tribunal de Rotterdam que una asociación que se dedicaba desde hacía 90 años a la protección de las aves, aunque no lo contuviese su Estatuto, absorbiera el interés general como interés propio ${ }^{36}$, lo cual le legitimó para recurrir ante los tribunales.

\subsection{El acto ilícito y los vecinos}

Sin perjuicio de todo lo dicho anteriormente en cuanto a los ruidos, su problemática y sus soluciones, es importante darse cuenta que la gran mayoría de los problemas sobre el ruido surgirá entre vecinos. Son famosas las peleas sobre los gallos y los perros, hasta tal grado que una cadena de la televisión neerlandesa dedicó un programa especial a los conflictos vecinales ${ }^{37}$.

34 Sentencia del Presidente del Departamento de Conflictos del Consejo del Estado de 19 de abril de 1988.

La ya citada Sentencia de 27 de junio de 1986.

36

Sentencia de marzo de 1991.

37

Buren, VPRO. 
Según el art. 5:37 Cc., los vecinos tienen el deber de no causarse molestias los unos a los otros, entendiendo por molestias entre otras el ruido. Sin embargo, este artículo por sí solo no otorga capacidad de acción a la víctima del ruido, sino que hace referencia al art. 6:162 Cc., el acto ilícito.

El acto ilícito contiene 5 elementos. El primer elemento se refiere a la definición de «acto ilícito», entendiendo por esto la infracción de un derecho, el actuar $\mathrm{u}$ omitir contrario a un deber legal y el actuar contrario a las costumbres y los usos sociales.

La infracción del Derecho se produce únicamente si se trata de una infracción de un derecho subjetivo absoluto, como es la propiedad y los derechos derivados de ella. Dentro de este marco es importante recordar que aún no existe un derecho subjetivo medioambiental, pero sí existe el derecho a un entorno vital sano.

La actuación u omisión contraria al deber legal, el segundo caso en que se presenta un acto ilícito, abarca también todas aquellas infracciones de normas establecidas en las licencias. En este sentido, queda claro que también el Ayuntamiento, o cualquier otro órgano de la Administración Pública, puede tener una acción derivante del acto ilícito contra un particular que no cumple las normas de la licencia ${ }^{38}$. Pero hay más. ¡También las actuaciones conformes a las reglas establecidas en la licencia pueden constituirse en un acto ilícito! Según explica el Tribunal Supremo, el hecho de que una determinada actividad tenga licencia no implica que esta actividad pierda su carácter ilícito. La licencia regula las relaciones entre las autoridades públicas y el poseedor de la licencia, pero no dicta las normas a cumplir por éste ante terceros afectados. Así, una persona que se dedica a la producción de cerezas, no tiene por qué aceptar los daños causados por la instalación de un vertedero junto a su terreno ${ }^{39}$. El daño en este caso residía en la preferencia de los cuervos y las gaviotas por las cerezas frescas en lugar de por los desperdicios del vertedero. Esta explicación del Tribunal Supremo se presta también a la aplicación de los problemas de ruido, que sufren los vecinos de discotecas, los vecinos de aeropuertos, etc.

Ahora bien, no todas las infracciones a un derecho o las actuaciones $u$ omisiones contrarias a un deber legal serán necesariamente consideradas un acto ilícito. En un lugar como los Países Bajos es imprescindible que se acepten determinadas molestias, de tal suerte que la existencia del acto ilícito depende también de las características, la duración y la gravedad de la molestia, tomando en cuenta el daño causado y las circunstancias de cada caso concreto, según

38 Sentencia de 20 de abril de 1981.

La famosa Sentencia de los cuervos de 10 de marzo de 1972. 
el Tribunal Supremo. Otro elemento para determinar la ilicitud de la actuación es la previsibilidad del daño. Así se consideró que el Estado cometió un acto ilícito promoviendo la instalación de una colonia de cormoranes en una región que se dedicaba a las piscifactorías ${ }^{40}$.

Los otros elementos que confluyen en el acto ilícito son la existencia de daño, la imputación al sujeto del acto ${ }^{41}$, y la causalidad entre el acto y el daño. El último elemento es la ya citada Schutznorm-theorie, dispuesta en el art. 6:163 Cc.

Las soluciones que el juez civil puede ofrecer son primeramente una indemnización, pero en los casos de ruido será más conveniente para la víctima conseguir una prohibición, eventualmente apoyada por una multa coercitiva. Aunque el art. 3:296 Cc. estipula la competencia del juez para imponer esta prohibición, el art. 6:168 Cc. exceptúa esta posibilidad si importantes intereses así lo exigen. Desde luego que es imposible paralizar el funcionamiento de todas las industrias que causan ruido, pero sí podrá imponerse la obligación de reducir los niveles de ruido al máximo, mediante medidas protectoras.

El último detalle peculiar a tratar es que las soluciones a que puede aspirar una asociación de protección medioambiental son más limitadas que las previstas para los particulares. El art. 3:305.a.3 Cc. dispone que una asociación de defensa de intereses generales no puede pretender la indemnización monetaria.

\section{CONSIDERACIÓN FINAL}

A lo largo de estas páginas se ha estudiado cómo se intenta solucionar el problema de la contaminación acústica en los Países Bajos, tanto en el Derecho administrativo como en el Derecho civil.

Se ha podido comprobar que se quiere lograr una integración de la legislación administrativa medioambiental, incluyendo también los problemas del ruido. Aunque ciertamente la Ley General de Medio Ambiente está en pleno desarrollo, aún quedan muchas materias fuera de su campo de aplicación, como en el caso del ruido son las zonas y las normas relativas a los aparatos, ambos instrumentos que todavía se encuentran regulados en la Ley de Molestias Acústicas.

\footnotetext{
40 Sentencia de 15 de febrero de 1991.

41 Sobre el problema de la culpa objetiva o subjetiva necesaria para determinar el acto ilícito me refiero al libro de C. DE Miguel PERALes, La responsabilidad civil por los daños al medio ambiente, Cívitas, Madrid, 1994.
} 
REALA 274-275 (MAYO-DICIEMBRE, 1997)

La Ley General de Medio Ambiente sí ha logrado otro objetivo de la política general del gobierno neerlandés: la retirada de la Administración de la vida empresarial e industrial. Mediante la aplicación de normas generales a cierto tipo de instalaciones, el número de licencias obligatorias ha disminuido ampliamente.

Pero quizá el mayor logro de la licencia consista en el hecho de que sea una licencia íntegra medioambiental, evitando de esta suerte el traslado de la contaminación a otros sectores. Al fin y al cabo, la naturaleza tampoco está compuesta por diferentes sectores, sino que es un conjunto en el que el hombre debe convivir con equilibrio.

\section{BIBLIOGRAFÍA}

C. DE Miguel Perales, La responsabilidad civil por daños al medio ambiente, Cívitas, Madrid, 1994.

P. NicolaII Y OTROS, Bestuursrecht, Factorum, Amsterdam, 1993.

L. Prakke y C. A. J. M. Kortmann, Het Staatsrecht van de landen der Europese Gemeenschappen, Kluwer, Deventer, 1988. 1992.

H. G. Sevenster, Milieubeleid en Gemeenschaprecht, Kluwer, Deventer,

F. P. C. L. TONNAER, Handboek van het Nederlandse Milieurecht, dos tomos, Uitgeverij LEMMA, Utrecht, 1994.

K. ZWEIGERT y H. KöTZ, An introduction to comparative law, 2." ed., traducción al inglés: T. Weir, Clarendon Paperbacks, Oxford, 1992. 


\section{Jurisprudencia}


